\title{
Association between dietary intake in each trimester during pregnancy and postpartum depression and hypochondriasis
}

\section{Maryam Azizi Kutenaee}

Hormozgan University of Medical Sciences

Seyed-Abdolvahab Taghavi

Yasuj University of Medical Sciences

Zatollah Asemi

Kashan University of Medical Sciences

Tahmineh Dadkhahtehrani

Isfahan University of Medical Sciences

Fatemeh Bazarganipour ( $\nabla$ f.bazarganipour@gmail.com )

Yasuj University of Medical Sciences

\section{Research}

Keywords: Nutrition, pregnancy, postpartum depression, hypochondriasis

Posted Date: May 29th, 2020

DOl: https://doi.org/10.21203/rs.3.rs-31266/v1

License: (c) (i) This work is licensed under a Creative Commons Attribution 4.0 International License. Read Full License 


\section{Abstract}

Background: The aim of the present study was to assess pregnant mothers' nutritional supply in each trimester and psychological status in postpartum with an emphasis on post pregnancy depression disorder and hypochondriasis.

Methods: This was a three-year prospective cohort study that 1319 pregnant women referred to rural health centers in Bandar Abbas, Iran were enrolled. The data were collected using a checklist including socioeconomic and fertility characterize, food frequency questionnaire (FFQ) for measurement of dietary intake, Edinburgh Postnatal Depression Scale (EPDS) to diagnose postpartum depression (PPD), Evans hypochondriasis questionnaire was used to diagnose hypochondriasis.

Results: The finding showed that prevalence of PPD and hypochondriasis were 91(6.8\%) and 553(42\%), respectively. The results showed that intake of iron, selenium, iodine, folate, vitamin C, B12, EPA in all trimesters and intake of vitamin $\mathrm{c}$ in second trimester was significantly associated with PPD $(P<0.05)$; as intake of those micronutrients was significantly lower in women with PPD rather than women without that. Moreover, our finding showed that intake of iron, iodine, folate, selenium, EPA in all trimesters was significantly associated with hypochondriasis $(P<0.05)$; as intake of those micronutrients was significantly lower in women with hypochondriasis rather than women without that.

Conclusions: Considering the pregnancy and lactation are major nutritional stressors to body and our finding in relation between poor nutrition intake of iron, iodine, folate, selenium, EPA, vitamin C and mood disorders (PPD and hypochondriasis), greeter attention to nutritional factors in psychological status during postpartum is warranted.

\section{Background}

Psychological disorders are among disabling dysfunctions all over the world and women in partum-post period are at higher risk for them [1]. Postnatal Depression Disorder (PDD) is characterized by sadness, fatigue, irritability, and lack of interest to daily activities [2]. Women suffering from PDD experience feelings like guilt, blame and worthlessness related to birth giving and becoming parent. This may lead to thinking of suicide or hurting the baby. According to the definition provided in Diagnostic and Statistical Manual of Mental Disorders (DSM -IV), PDD begins during four weeks after giving birth [2]. This disorder often remains undiagnosed and uncured due to unawareness of signs and the related stigma [3], and as a result it seems essential to take necessary measurements to diagnose risk factors.

Hypochondriasis or illness anxiety disorder is a disorder that the suffering person considers himself/herself as patient despite the fact that there is no health problem shown in comprehensive medical examinations $[4,5]$. Hypochondriasis could result in problems in daily activity and social relations (due to the patient's expectation of being cured and being paid special attention) and also in occupational failure [6]. Researchers have shown a relation between depression and hypochondriasis [7, 8]; but there is a lack of studies on the prevalence and related factors of hypochondriasis during the 
pregnancy and postpartum. For now, the precise etiology of PDD and hypochondriasis is unknown; but it is generally believed that biological, genetic, hormonal, and psychological factors are related with them [9-16]. Nutrients like folic acid, vitamin B12, calcium, zinc, selenium, iron, non-saturated greasy acids are considered as significant factors $[11,14,17]$. Nutrition has been proven to play effective role in preventing mental health disorders; the roles include engaging in synthesis and balancing neurotransmitters and catecholamines, activity of enzymes, cellular and oxidative process, function of receptors ,preserving neural tissue and neural functions which are of great significance in mental health[18, 19].

Pregnant mothers are more vulnerable due to the considerable pressures received by hormonal, metabolic, and physical changes that increase their nutritional needs [20]. Also, nutritional deficiencies due to breast feeding and delay postpartum repletion are more significant in post-partum period and should be paid more attention. Nutrition, in addition to its impact on mental health, have considerable importance, since it can be improved. In developing countries including Iran, women living in disadvantaged and deprived areas are at more risk regarding nutrition. In these regions, sex discrimination has given the women of family the lowest priority for feeding; their foods have not only the lowest quantity but the poorest quality. Hormozgan is one of the disadvantaged and deprived southern provinces in Iran where no studies regarding receiving micronutrients have ever done. According to account of Nutrition Improvement Office of Ministry of health and Treatment of Iran, Hormozgan is one of five provinces in Iran which has an undesirable situation about malnutrition and nutritious safety and the shortage of micronutrients, iron, vitamins within vulnerable groups, particularly pregnant women, is increasing. Unfortunately, there is no study conducted to examine the association between nutritional supply within each trimester and mental status in postpartum period. Considering the importance of public psychological health, the fact that there is no effective treatment offered yet, and regarding abovementioned issues, this research has been designed and conducted to assess pregnant mothers' nutritional supply in each trimester and psychological status in postpartum with an emphasis on postpartum depression disorder and hypochondriasis.

\section{Materials And Methods}

\section{Design and data collection}

This is a perspective cohort study in 2015-2017 on pregnant women referring to health centers of Bandar-Abbas, Iran. Inclusion criteria were willing to participating in study, single pregnancy, gestational age less than 10 weeks, age 15-40 years, being married, primagravida mother, Iranian nationality, having no history of systemic diseases (diabetes, hypertension, known anemia, and other diseases requiring special diet) before pregnancy, no smoking or drinking alcohol. Exclusion criteria were unwilling to continue the study. Data were collected using a questionnaire. After explaining the objectives of the study and giving assurance about confidentiality of information, written consent forms were obtained. All questionnaires were completed by well-trained researchers. Convenience sampling was used and all pregnant women with inclusion criteria entered the study. The interval of prenatal visits and cares were consistent with National Guideline of Prenatal Care in Iran, as seven visits were given in weeks of 6-10, 
16-20, 26-30, 31-34, 35-37, 38-39, and 41 respectively. Dose of iron, folic acid, and multivitamins was recorded precisely all over the pregnancy period. There are no significant different between total dose of iron, folic acid, and multivitamins during pregnant between depression vs. non depressed and hypochondriac vs. non hypochondriac (data not presented).

\section{Measure}

1. 1. A check-list was used to collect demographic information (age, education of woman and her husband, job of woman and her husband), socioeconomic status, and BMI.

2. 2. Edinburgh Postnatal Depression Scale (EPDS) was used to diagnose postnatal depression. It was developed by Scottish health centers in Edinburgh and Livingston in 1978 and composed of 10 short statements. Each answer is given a score of 0 to 3 based on Likert scale. The total score is $0-30$. A score of more than 12 shows depression [21]. The validity and reliability of this questionnaire is approved in Iran [22]. The questionnaire was completed during 4-6 weeks after giving birth.

3. 3. Evans hypochondriasis questionnaire was used to diagnose hypochondriasis. It was developed by Evans et al in 1978 and composed of 36 questions [23]. Some of them have two options and the others have 5 options. The total score is $0-60$. The score of $0-20,21-40,41-60$ indicate lack of mild hypochondriasis, moderate hypochondriasis; sever hypochondriasis, mild hypochondriasis, moderate hypochondriasis, severe hypochondriasis. The validity and reliability of this questionnaire is approved in Iran $[7,24]$. The questionnaire was completed during $4-6$ weeks after giving birth.

4. 4. Food frequency questionnaire: dietary intake was measured using a modified food frequency questionnaire (FFQ) based on Iranian dietary questionnaire which contains 168 items. The reliability and validity of the questionnaire are approved in Iran [25]. FFQ included a list of foods with a standard size of a food. Subjects were asked to report the frequency of consumption of each food during the past month on a daily, weekly or monthly basis. The amount of nutritional item consumed was converted to grams using household scales. This dietary information was analyzed using the software Nutrition4 which calculated the amount of energy, macronutrients (carbohydrates, lipid, and protein) and micronutrients (at least 30 micronutrients) including fat soluble vitamins, water soluble vitamins and minerals. The FFQ was completed in the first of each trimester of pregnancy.

\section{Ethics}

The study protocol was approved by the ethics review committee of Hormozgan University of medical sciences. Participation was voluntary and they were permitted to discontinue their cooperation. All questionnaires were anonymous and the researcher assured the participants of confidentiality of information. After explaining the purposes of the study, all of them signed a written consent form.

\section{Statistical analysis}

Descriptive statistics was used to summarize the quantitative and qualitative variables. Data is presented as mean (standard division) for quantitative variables and number (percent) for qualitative. Analytic 
statistics including T-test (for variables with normal distribution based on Kolmogorov-Smirnov test), chi square, and Mann-Whitney were used for inter-group comparison of variables. Food frequency questionnaires were analyzed applying software N4. Other data were analyzed using SPSS 21 (SPSS, Chicago, IL, USA). $\mathrm{P}<0.05$ was considered as significant level.

\section{Results}

\section{The study samples}

In a three-year period, 1319 women completed full course of the study. Socioeconomic and reproductive characteristics are summarized in Table 1. The results showed that $91(6.8 \%)$ women had PPD and $553(42 \%)$ had hypochondriasis.

Table 1

Demographic and clinical characterizes of participants

\begin{tabular}{|lll|}
\hline Age * & \multicolumn{2}{l}{$\mathbf{2 4 . 3 4} \pm \mathbf{5 . 5 5}$} \\
\hline Age of husbend * & $28.16 \pm 6.24$ \\
\hline Occupation** & Houswife & $1289(96.9)$ \\
\cline { 2 - 3 } & Employed & $41(3.1)$ \\
\hline Education * & $9.27 \pm 4.14$ \\
\hline Gestational age* & $171.49 \pm 114.67$ \\
\hline BMl* & $23.07 \pm 29.29$ \\
\hline Hb in 6-10 weeks of pregnancy* & $12.79 \pm 0.12$ \\
\hline HCT in 6-10 weeks of pregnancy* & $35.78 \pm 4.32$ \\
\hline$*$ mean \pm SD & \\
\hline$* \star N(\%)$ & \\
\hline
\end{tabular}


Table 2

Prevlance of PPD and hypochondriasis

\begin{tabular}{|lll|}
\hline Depression * & No & $1239(93.2)$ \\
\cline { 2 - 3 } Hypochondriasis* & Yes & $91(6.8)$ \\
& No & $766(57.6)$ \\
\cline { 2 - 3 } & Marginal & $366(27.5)$ \\
\cline { 2 - 3 } & Mild & $99(7.4)$ \\
\cline { 2 - 3 } & Moderate & $56(4.2)$ \\
\cline { 2 - 3 } & Severe & $10(0.08)$ \\
\hline \multirow{2}{*}{$\mathrm{N}(\%)$} & & \\
\hline
\end{tabular}

\section{Relation Between Dietary Intake And Hypochondriasis}

The results showed that intake of iron, iodine, folate, selenium, EPA in all trimesters was significantly associated with hypochondriasis $(p<0.05)$; as intake of those micronutrients was significantly lower in women with hypochondriasis rather than women without that (Table 3-5). 
Table 3

Comparison of dietary intake in participants based on hypochondriac status in the first trimester

\begin{tabular}{|c|c|c|c|}
\hline Variable ** & $\begin{array}{l}\text { No problem } \\
(n=766)\end{array}$ & $\begin{array}{l}\text { Problem } \\
(n=553)\end{array}$ & $P$ value * \\
\hline Energy (Kcal/day) & $1501.41 \pm 96.29$ & $1443.67 \pm 95.36$ & 0.23 \\
\hline Protein (\% energy) & $88.35 \pm 64.85$ & $82.82 \pm 61.87$ & 0.39 \\
\hline Fat (\% energy) & $87.06 \pm 18.22$ & $83.35 \pm 15.55$ & 0.58 \\
\hline Saturated fatty acids (g) & $26.72 \pm 33.97$ & $25.01 \pm 24.17$ & 0.58 \\
\hline Polyunsaturated fatty acids (g) & $24.16 \pm 14.17$ & $23.55 \pm 18.58$ & 0.52 \\
\hline Linoleic acids (g) & $22.46 \pm 22.11$ & $21.71 \pm 17.38$ & 0.21 \\
\hline EPA (g) & $0.03 \pm 0.002$ & $0.001 \pm 0.001$ & 0.001 \\
\hline Sodium (mg/day) & $1293.54 \pm 98.95$ & $1004.94 \pm 15.77$ & 0.06 \\
\hline Iron (mg/day) & $49.20 \pm 3.78$ & $27.80 \pm 6.13$ & 0.001 \\
\hline Magnesium (mg/day) & $504.31 \pm 38.26$ & $489.90 \pm 26.88$ & 0.84 \\
\hline Zinc (mg/day) & $13.35 \pm 3.75$ & $12.86 \pm 2.55$ & 0.59 \\
\hline Manganese (mg/day) & $8.81 \pm 6.72$ & $8.77 \pm 7.89$ & 0.98 \\
\hline Fluoride $(\mu \mathrm{g} /$ day $)$ & $1669.37 \pm 77.73$ & $1460.16 \pm 75.59$ & 0.43 \\
\hline lodine ( $\mu \mathrm{g} /$ day) & $0.29 \pm 0.42$ & $0.00 \pm 0.00$ & 0.001 \\
\hline Vitamin A ( $\mu \mathrm{g} /$ day) & $1532.33 \pm 44.50$ & $1250.79 \pm 63.69$ & 0.47 \\
\hline Vitamin E (mg/day) & $8.92 \pm 7.98$ & $7.96 \pm 9.25$ & 0.42 \\
\hline Vitamin B1 (mg/day) & $2.14 \pm 3.59$ & $1.91 \pm 1.36$ & 0.33 \\
\hline Vitamin B3 (mg/day) & $26.05 \pm 23.95$ & $25.74 \pm 18.97$ & 0.85 \\
\hline Folate ( $\mu \mathrm{g} /$ day) & $483.04 \pm 82.27$ & $461.59 \pm 47.82$ & 0.04 \\
\hline Carbohydrate (g/day) & $380.26 \pm 54.02$ & $322.02 \pm 10.13$ & 0.60 \\
\hline Calcium (mg/day) & $1234.81 \pm 12.91$ & $1161.700 \pm 16.69$ & 0.81 \\
\hline Phosphorus (mg/day) & $1800.16 \pm 10.80$ & $1775.22 \pm 79.80$ & 0.53 \\
\hline Selenium (mg/day) & $1.78 \pm 0.05$ & $0.18 \pm 0.06$ & 0.03 \\
\hline Vitamin C (mg/day) & $400.60 \pm 12.18$ & $359.47 \pm 62.52$ & 0.33 \\
\hline
\end{tabular}

*T test, ** Mean \pm SD 


\begin{tabular}{|llll|}
\hline Variable ** & $\begin{array}{l}\text { No problem } \\
(\mathbf{n}=766)\end{array}$ & $\begin{array}{l}\text { Problem } \\
(\mathbf{n}=\mathbf{5 5 3})\end{array}$ & P value * \\
\hline Vitamin B12 $(\mu \mathrm{g} /$ day $)$ & $5.40 \pm 0.58$ & $3.82 \pm 7.89$ & 0.05 \\
\hline$*$ T test, $* \star$ Mean \pm SD & & & \\
\hline
\end{tabular}


Table 4

Comparison of dietary intake in participants based on hypochondriac status in the second trimester

\begin{tabular}{|c|c|c|c|}
\hline Variable ** & $\begin{array}{l}\text { No problem } \\
(n=766)\end{array}$ & $\begin{array}{l}\text { Problem } \\
(n=553)\end{array}$ & $P$ value * \\
\hline Energy (Kcal/day) & $1776.64 \pm 64.03$ & $1541.72 \pm 96.29$ & 0.20 \\
\hline Protein (\% energy) & $134.93 \pm 60.68$ & $107.43 \pm 58.53$ & 0.24 \\
\hline Fat (\% energy) & $104.00 \pm 54.53$ & $102.53 \pm 70.70$ & 0.88 \\
\hline Saturated fatty acids (g) & $27.71 \pm 33.21$ & $21.72 \pm 42.35$ & 0.20 \\
\hline Polyunsaturated fatty acids (g) & $27.41 \pm 25.13$ & $26.60 \pm 35.58$ & 0.73 \\
\hline Linoleic acids (g) & $25.47 \pm 23.06$ & $24.13 \pm 30.83$ & 0.52 \\
\hline EPA (g) & $0.34 \pm 3.93$ & $0.01 \pm 0.01$ & 0.001 \\
\hline Sodium (mg/day) & $1773.88 \pm 91.69$ & $1042.43 \pm 93.56$ & 0.34 \\
\hline Iron (mg/day) & $29.14 \pm 27.82$ & $23.81 \pm 23.51$ & 0.03 \\
\hline Magnesium (mg/day) & $672.52 \pm 91.80$ & $612.74 \pm 68.77$ & 0.18 \\
\hline Zinc (mg/day) & $22.06 \pm 12.66$ & $13.67 \pm 19.19$ & 0.13 \\
\hline Manganese (mg/day) & $9.30 \pm 3.69$ & $8.10 \pm 7.08$ & 0.18 \\
\hline Fluoride ( $\mu \mathrm{g} /$ day) & $1871.62 \pm 20.08$ & $1513.33 \pm 99.90$ & 0.30 \\
\hline lodine ( $\mu \mathrm{g} /$ day) & $1.72 \pm 5.07$ & $0.07 \pm 0.68$ & 0.001 \\
\hline Vitamin A ( $\mu \mathrm{g} /$ day) & $1998.46 \pm 57.64$ & $1455.59 \pm 39.94$ & 0.20 \\
\hline Vitamin E (mg/day) & $57.76 \pm 32.02$ & $11.31 \pm 18.78$ & 0.17 \\
\hline Vitamin B1 (mg/day) & $3 \pm 9.70$ & $2.15 \pm 2.42$ & 0.17 \\
\hline Vitamin B3 (mg/day) & $39.13 \pm 43.13$ & $31.29 \pm 40.62$ & 0.39 \\
\hline Folate ( $\mu \mathrm{g} /$ day) & $889.21 \pm 20.23$ & $553.92 \pm 86.17$ & 0.02 \\
\hline Carbohydrate (g/day) & $579.72 \pm 34.54$ & $450.72 \pm 90.11$ & 0.29 \\
\hline Calcium (mg/day) & $1921.06 \pm 53.34$ & $1481.06 \pm 80.02$ & 0.40 \\
\hline Phosphorus (mg/day) & $1800.16 \pm 10.80$ & $1075.22 \pm 79.80$ & 0.53 \\
\hline Selenium (mg/day) & $0.19 \pm 0.16$ & $0.03 \pm 0.05$ & 0.01 \\
\hline Vitamin C (mg/day) & $444.73 \pm 32.15$ & $236.98 \pm 32.38$ & 0.23 \\
\hline
\end{tabular}

*T test, **Mean \pm SD 


\begin{tabular}{|llll|}
\hline Variable ** & $\begin{array}{l}\text { No problem } \\
(\mathbf{n}=\mathbf{7 6 6})\end{array}$ & $\begin{array}{l}\text { Problem } \\
(\mathbf{n}=\mathbf{5 5 3})\end{array}$ & P value * \\
\hline Vitamin B12 $(\mu \mathrm{g} /$ day $)$ & $3.42 \pm 0.45$ & $2.93 \pm 4.22$ & 0.32 \\
\hline *T test, **Mean \pm SD & & & \\
\hline
\end{tabular}


Table 5

Comparison of dietary intake in participants based on hypochondriac status in the third trimester

\begin{tabular}{|c|c|c|c|}
\hline Variable ** & $\begin{array}{l}\text { No problem } \\
(n=766)\end{array}$ & $\begin{array}{l}\text { Problem } \\
(n=553)\end{array}$ & $P$ value * \\
\hline Energy (Kcal/day) & $1481.37 \pm 96.16$ & $1376.51 \pm 84.12$ & 0.20 \\
\hline Protein (\% energy) & $86.94 \pm 89.09$ & $78.38 \pm 28.29$ & 0.24 \\
\hline Fat (\% energy) & $89.58 \pm 14.15$ & $71.40 \pm 10.65$ & 0.28 \\
\hline Saturated fatty acids (g) & $57.93 \pm 58.14$ & $55.45 \pm 37.13$ & 0.48 \\
\hline Polyunsaturated fatty acids (g) & $25.92 \pm 29.18$ & $21.04 \pm 26.36$ & 0.92 \\
\hline Linoleic acids (g) & $23.45 \pm 6.43$ & $19.37 \pm 5.23$ & 0.21 \\
\hline EPA (g) & $0.02 \pm 0.000$ & $0.01 \pm 0.001$ & 0.001 \\
\hline Sodium (mg/day) & $3000.43 \pm 98.86$ & $2098.90 \pm 71.0$ & 0.06 \\
\hline Iron (mg/day) & $34.99 \pm 2.53$ & $21.82 \pm 2.49$ & 0.04 \\
\hline Magnesium (mg/day) & $342.87 \pm 73.90$ & $388.60 \pm 74.83$ & 0.51 \\
\hline Zinc (mg/day) & $14.08 \pm 4.68$ & $11.17 \pm 4.01$ & 0.59 \\
\hline Manganese (mg/day) & $9.99 \pm 12.61$ & $7.14 \pm 5.54$ & 0.48 \\
\hline Fluoride ( $\mu \mathrm{g} /$ day) & $1996.06 \pm 63.84$ & $1278.21 \pm 25.61$ & 0.62 \\
\hline lodine ( $\mu \mathrm{g} /$ day) & $1.02 \pm 1.14$ & $0.08 \pm 0.42$ & 0.05 \\
\hline Vitamin A ( $\mu \mathrm{g} /$ day) & $1977.34 \pm 68.04$ & $1790.51 \pm 43.01$ & 0.39 \\
\hline Vitamin E (mg/day) & $10.78 \pm 16.22$ & $7.37 \pm 11.99$ & 0.92 \\
\hline Vitamin B1 (mg/day) & $3.27 \pm 5.65$ & $1.77 \pm 2.27$ & 0.35 \\
\hline Vitamin B3 (mg/day) & $38.46 \pm 6.68$ & $27.17 \pm 5.57$ & 0.33 \\
\hline Folate ( $\mu \mathrm{g} /$ day) & $883.88 \pm 69.60$ & $432.60 \pm 9.96$ & 0.02 \\
\hline Carbohydrate (g/day) & $654.94 \pm 13.12$ & $336.22 \pm 19.42$ & 0.60 \\
\hline Calcium (mg/day) & $1336.72 \pm 57.15$ & $1136.97 \pm 34.66$ & 0.51 \\
\hline Phosphorus (mg/day) & $1522.04 \pm 46.40$ & $1497.83 \pm 17.93$ & 0.08 \\
\hline Selenium (mg/day) & $0.26 \pm 0.01$ & $0.09 \pm 0.002$ & 0.01 \\
\hline Vitamin C (mg/day) & $302.25 \pm 17.20$ & $270.93 \pm 13.84$ & 0.65 \\
\hline
\end{tabular}

*T test, **Mean \pm SD 


\begin{tabular}{|llll|}
\hline Variable ** & $\begin{array}{l}\text { No problem } \\
(\mathbf{n}=766)\end{array}$ & $\begin{array}{l}\text { Problem } \\
(\mathbf{n}=\mathbf{5 5 3})\end{array}$ & P value * \\
\hline Vitamin B12 $(\mu \mathrm{g} /$ day $)$ & $3.65 \pm 0.42$ & $3.02 \pm 0.63$ & 0.84 \\
\hline$*$ T test, $*$ Mean \pm SD & & & \\
\hline
\end{tabular}

\section{Relation Between Dietary Intake And Ppd}

The results showed that intake of iron, selenium, iodine, folate, vitamin C, B12, EPA in all trimesters $(p<$ $0.005)$ and intake of vitamin $c$ in second trimester was significantly associated with PPD $(p<0.005)$; as intake of those micronutrients was significantly lower in women with PPD rather than women without that (Table 6-8). 
Table 6

Comparison of dietary intake in participants based on PPD status in the first trimester

\begin{tabular}{|c|c|c|c|}
\hline Variable ** & $\begin{array}{l}\text { No problem } \\
(n=1228)\end{array}$ & $\begin{array}{l}\text { Problem } \\
(n=91)\end{array}$ & $P$ value* \\
\hline Energy (Kcal/day) & $1576.14 \pm 52.57$ & $1223.36 \pm 27.52$ & 0.73 \\
\hline Protein (\% energy) & $101.66 \pm 46.24$ & $79.84 \pm 61.58$ & 0.39 \\
\hline Fat (\% energy) & $105.05 \pm 22.50$ & $92.33 \pm 93.31$ & 0.58 \\
\hline Saturated fatty acids (g) & $33.94 \pm 23.84$ & $27.36 \pm 45.81$ & 0.56 \\
\hline Polyunsaturated fatty acids (g) & $24.59 \pm 23.58$ & $22.12 \pm 17.96$ & 0.32 \\
\hline Linoleic acids (g) & $22.46 \pm 21.61$ & $20.46 \pm 16.76$ & 0.38 \\
\hline EPA (g) & $0.02 \pm 0.02$ & $0.01 \pm 0.01$ & 0.01 \\
\hline Sodium (mg/day) & $1910.41 \pm 48.05$ & $1588.59 \pm 12.65$ & 0.80 \\
\hline Iron (mg/day) & $32.74 \pm 82.03$ & $18.30 \pm 13.11$ & 0.05 \\
\hline Magnesium (mg/day) & $314.60 \pm 87.07$ & $326.92 \pm 80.19$ & 0.10 \\
\hline Zinc (mg/day) & $13.54 \pm 29.71$ & $9.46 \pm 8.96$ & 0.19 \\
\hline Manganese (mg/day) & $8.99 \pm 7.96$ & $7.59 \pm 3.42$ & 0.90 \\
\hline Fluoride ( $\mu \mathrm{g} /$ day) & $1667.38 \pm 41.42$ & $1167.69 \pm 42.49$ & 0.19 \\
\hline lodine ( $\mu \mathrm{g} /$ day) & $0.00 \pm 0.00$ & $0.03 \pm 0.00$ & $<0.001$ \\
\hline Vitamin A ( $\mu \mathrm{g} /$ day) & $1708.65 \pm 31.29$ & $1537.65 \pm 56.32$ & 0.16 \\
\hline Vitamin E (mg/day) & $9.006 \pm 17.26$ & $5.28 \pm 4.17$ & 0.07 \\
\hline Vitamin B1 (mg/day) & $2.14 \pm 3.40$ & $1.56 \pm 1.11$ & 0.10 \\
\hline Vitamin B3 (mg/day) & $26.21 \pm 23.58$ & $23.02 \pm 15.14$ & 0.20 \\
\hline Folate ( $\mu \mathrm{g} /$ day) & $490.98 \pm 52.89$ & $346.95 \pm 50.62$ & 0.05 \\
\hline Carbohydrate (g/day) & $829.13 \pm 52.57$ & $696.53 \pm 65.24$ & 0.40 \\
\hline Calcium (mg/day) & $1221.32 \pm 19.20$ & $983.74 \pm 40.73$ & 0.20 \\
\hline Phosphorus (mg/day) & $1538.73 \pm 437.73$ & $1229.32 \pm 19.20$ & 0.34 \\
\hline Selenium (mg/day) & $0.48 \pm 1.43$ & $0.13 \pm 0.36$ & $<0.001$ \\
\hline Vitamin C (mg/day) & $337.87 \pm 87.89$ & $337.46 \pm 40.21$ & 0.30 \\
\hline Vitamin B12 ( $\mu \mathrm{g} /$ day) & $63.59 \pm 55.14$ & $34.20 \pm 20.63$ & 0.05 \\
\hline${ }^{\star T}$ T test, **Mean \pm SD & & & \\
\hline
\end{tabular}


Table 7

Comparison of dietary intake in participants based on PPD status in the second trimester

\begin{tabular}{|llll|}
\hline Variable ** & $\begin{array}{l}\text { No problem } \\
(\mathbf{n}=1239)\end{array}$ & $\begin{array}{l}\text { Problem } \\
(\mathbf{n}=91)\end{array}$ & P value * \\
\hline Energy (Kcal/day) & $1492.88 \pm 816.00$ & $1157.72 \pm 110.10$ & 0.15 \\
\hline Protein (\% energy) & $133.22 \pm 43.52$ & $84.59 \pm 11.01$ & 0.17 \\
\hline Fat (\% energy) & $105.37 \pm 150.00$ & $81.57 \pm 95.33$ & 0.13 \\
\hline Saturated fatty acids (g) & $66.67 \pm 80.33$ & $22.34 \pm 28.39$ & 0.40 \\
\hline Polyunsaturated fatty acids (g) & $27.32 \pm 34.58$ & $19.07 \pm 21.27$ & 0.82 \\
\hline Linoleic acids (g) & $24.88 \pm 34.58$ & $17.55 \pm 19.58$ & 0.28 \\
\hline EPA (g) & $0.30 \pm 3.63$ & $0.009 \pm 0.01$ & 0.001 \\
\hline Sodium (mg/day) & $1895.04 \pm 21.19$ & $1860.60 \pm 58.61$ & 0.50 \\
\hline Iron (mg/day) & $28.27 \pm 53.57$ & $16.65 \pm 43.72$ & 0.04 \\
\hline Magnesium (mg/day) & $543.20 \pm 15.56$ & $474.47 \pm 95.77$ & 0.43 \\
\hline Zinc (mg/day) & $47.33 \pm 77.16$ & $13.34 \pm 21.33$ & 0.39 \\
\hline Manganese (mg/day) & $9.16 \pm 12.94$ & $7.96 \pm 9.95$ & 0.38 \\
\hline Fluoride ( $\mu$ g/day) & $1727.13 \pm 41.25$ & $1642.49 \pm 58.20$ & 0.08 \\
\hline lodine ( $\mu$ g/day) & $4.38 \pm 0.91$ & $0.00 \pm 0.00$ & $<0.001$ \\
\hline Vitamin A ( $\mu$ g/day) & $1556.34 \pm 64.52$ & $1099.25 \pm 42.84$ & 0.56 \\
\hline Vitamin E (mg/day) & $12.25 \pm 8.26$ & $8.11 \pm 10.64$ & 0.39 \\
\hline Vitamin B1 (mg/day) & $2.91 \pm 9.11$ & $2.04 \pm 2.97$ & 0.36 \\
\hline Vitamin B3 (mg/day) & $38.31 \pm 34.67$ & $29.30 \pm 43.99$ & 0.52 \\
\hline Folate ( $\mu$ g/day) & $550.03 \pm 56.86$ & $522.57 \pm 34.42$ & 0.43 \\
\hline Carbohydrate (g/day) & $589.11 \pm 0.16$ & $569.51 \pm 20.33$ & 0.93 \\
\hline Calcium (mg/day) & $1591.66 \pm 29.43$ & $1436.16 \pm 27.13$ & 0.41 \\
\hline Phosphorus (mg/day) & $1851.02 \pm 33.46$ & $1795.43 \pm 30.13$ & 0.28 \\
\hline Selenium (mg/day) & $1.60 \pm 0.06$ & $0.54 \pm 1.69$ & 0.05 \\
\hline Vitamin C (mg/day) & $449.26 \pm 80.01$ & $305.09 \pm 16.83$ & 0.04 \\
\hline
\end{tabular}

*T test, ${ }^{*}$ Mean \pm SD 


\begin{tabular}{|llll|}
\hline Variable ** & $\begin{array}{l}\text { No problem } \\
(\mathbf{n}=\mathbf{1 2 3 9})\end{array}$ & $\begin{array}{l}\text { Problem } \\
(\mathbf{n}=\mathbf{9 1})\end{array}$ & P value * \\
\hline Vitamin B12 ( $\mathrm{gg} /$ day $)$ & $118.92 \pm 96.14$ & $90.76 \pm 54.96$ & 0.05 \\
\hline *T test, **Mean \pm SD & & & \\
\hline
\end{tabular}


Table 8

Comparison of dietary intake in participants based on PPD status in the third trimester

\begin{tabular}{|llll|}
\hline Variable ** & $\begin{array}{l}\text { No problem } \\
(\mathbf{n}=1239)\end{array}$ & $\begin{array}{l}\text { Problem } \\
(\mathbf{n}=91)\end{array}$ & P value * \\
\hline Energy (Kcal/day) & $1501.31 \pm 78.45$ & $1478.67 \pm 90.12$ & 0.09 \\
\hline Protein (\% energy) & $106.85 \pm 28.06$ & $96.38 \pm 21.11$ & 0.09 \\
\hline Fat $(\%$ energy) & $103.98 \pm 73.78$ & $97.30 \pm 45.15$ & 0.58 \\
\hline Saturated fatty acids (g) & $32.36 \pm 78.88$ & $18.95 \pm 34.16$ & 0.10 \\
\hline Polyunsaturated fatty acids $(\mathrm{g})$ & $22.64 \pm 27.37$ & $12.56 \pm 18.33$ & 0.36 \\
\hline Linoleic acids (g) & $22.77 \pm 14.53$ & $20.82 \pm 26.23$ & 0.27 \\
\hline EPA (g) & $0.02 \pm 0.02$ & $0.009 \pm 0.01$ & $<0.001$ \\
\hline Sodium (mg/day) & $1534.62 \pm 81.65$ & $1296.77 \pm 51.22$ & 0.45 \\
\hline Iron (mg/day) & $24.61 \pm 29.70$ & $19.27 \pm 42.36$ & 0.05 \\
\hline Magnesium (mg/day) & $390.95 \pm 73.52$ & $220.47 \pm 38.92$ & 0.11 \\
\hline Zinc (mg/day) & $13.80 \pm 26.50$ & $10.11 \pm 23.20$ & 0.19 \\
\hline Manganese (mg/day) & $7.93 \pm 7.48$ & $4.11 \pm 5.58$ & 0.68 \\
\hline Fluoride ( $\mu \mathrm{g} /$ day) & $1242.08 \pm 61.40$ & $1084.72 \pm 93.16$ & 0.78 \\
\hline Iodine $(\mu \mathrm{g} /$ day) & $0.39 \pm 0.85$ & $0.00 \pm 0.00$ & $<0.001$ \\
\hline Vitamin A ( $\mu \mathrm{g} /$ day) & $1155.61 \pm 76.08$ & $1083.79 \pm 69.34$ & 0.36 \\
\hline Vitamin E (mg/day) & $6.29 \pm 3.29$ & $4.05 \pm 5.24$ & 0.87 \\
\hline Vitamin B1 (mg/day) & $2.10 \pm 3.29$ & $1.22 \pm 2.14$ & 0.74 \\
\hline Vitamin B3 (mg/day) & $29.45 \pm 34.51$ & $26.52 \pm 63.24$ & 0.53 \\
\hline Folate $(\mu \mathrm{g} /$ day) & $510.56 \pm 95.49$ & $379.95 \pm 65.56$ & 0.05 \\
\hline Carbohydrate (g/day) & $308.97 \pm 70.39$ & $205.64 \pm 38.14$ & 0.81 \\
\hline Calcium (mg/day) & $611.08 \pm 47.56$ & $522.67 \pm 82.77$ & 046 \\
\hline Phosphorus (mg/day) & $1228.24 \pm 81.26$ & $1048.83 \pm 80.50$ & 0.63 \\
\hline Selenium (mg/day) & $4.56 \pm 0.46$ & $2.28 \pm 2.39$ & 0.05 \\
\hline Vitamin C (mg/day) & $362.68 \pm 30.25$ & $184.65 \pm 54.36$ & 0.75 \\
\hline Vitamin B12 ( $\mu$ g/day) & $3.45 \pm 1.58$ & $1.29 \pm .032$ & 0.02 \\
\hline$*$ test, **Mean \pm SD & & & \\
\hline
\end{tabular}




\section{Discussion}

The present study as the first study in world aimed to assess the association of PPD and postpartum hypochondriasis with intake of micronutrients in each trimester. The results showed that intake of iron, selenium, iodine, folate, vitamin C, B12, EPA in all trimesters and vitamin c in second trimester was significantly lower in women with PPD rather than women without that.

Iron as the main component of hemoglobin of red blood cells is responsible for transporting oxygen. Iron deficiency induces anemia and results in insufficient oxygen delivery to cells [26, 27]. Also, Iron deficiency anemia with decline of hemoglobin concentration increased significantly risk of depression in an independent dose pattern among old population; as, the lower the level of hemoglobin the worse the severity of depression [28]. Further studies in future are needed to determine the optimal and safe dose of iron supplements for depressive pregnant women.

Regarding selenium, recent studies showed depression symptoms in patients with low level of serum selenium that were treated and improved with selenium supplements [29]. Another study showed that selenium supplements during pregnancy declined depression (based on score of Edinburg questionnaire) in 8 weeks post-partum [30]. It is unknown the mechanism of impact of selenium on mood. Selenium is necessary for synthesis and metabolism of thyroid hormones. Little changes in function of thyroid are associated with depression [31]. Selenium deficiency causes thyroid hormone dysfunction that may mediates impact of selenium on depression [31]. Selenium is a main component of antioxidant enzyme glutathione peroxidase that is involved in antioxidant mechanisms and protects nerves of damages and lipoperoxidation [19]. Based on a randomized clinical trial in patients with HIV, the researchers reported that selenium supplement improved depressive mood and quality of life score 20 times [32]. Also, selenium supplement (100-150 microgram per day) for 5-6 weeks improved depressed-dejected mood state and a trend toward improvement in quality of life scores [32] .

Interaction between selenium and iodine in synthesis of thyroid hormones is a significant concern in Iran due to selenium and iodine sufficiency. lodine sufficiency is a health problem in Iran. After performing the mandatory fortification program in 1991, many children and adults have optimal iodine intake [33]. Unfortunately, the present study showed that insufficient intake and iodine deficiency continues in pregnant women despite implement of mandatory iodine fortification. Selenium deficiency could worsen the consequences of iodine deficiency among this vulnerable group [34]. lodine is a trace element that is an essential component of thyroid hormones It was shown that hypothyroidism is associated with mood disorders [35, 36], cognitive and affective disturbances [37] and memory impairment [38]. Also the relationship between depression and subliclinical hypothyroidism in animal model was investigated [39].

The present study showed that insufficient intake of some antioxidants is associated with PPD. Antioxidants (vitamin C/ B12, Folate) could be protective as a defense mechanism against PPD [40]. Antioxidants (vitamin C/ B12, Folate) could be protective as a defense mechanism against brain/vascular damages of oxidative stress related to reactive oxygen species (ROS) [40]. Folate and vitamin B12 have fundamental roles in normal function of central nervous system and could regulate 
mood through some mechanisms [40]. For example, they are essential for some metabolic process in CNS such as methylation and construction of serotonin and other neurotransmitters, neuroamines, and catecholamines [41, 42]. Also, metabolism of hemosystein depends on sufficient levels of folate and vitamin B12 and the level of hemosystein is a sensitive indicator of deficiency of folate and vitamin B12 [43]. Based on some studies, level of hemosystein is higher and level of vitamin B12 and folate is lower in depressive patients [44]. Higher level of hemosystein could induce a vascular response resulting in depression [45]. A recent meta-analysis showed that folate deficiency is associated with a $55 \%$ increased risk of depression disregarding folic acid fortification or method of assessment of folate intake (questionnaire/serum folate measurement) [46]. Also, it was determined that the patients with lower level of folate had weaker response to antidepressants $[47,48]$. Several studies indicated improvement of depression symptoms after adding folic acid to antidepressants [49-52]. Previous studies reported that high intake of vitamin B12, selenium, and magnesium was associated with lower depression syndromes in pregnant and non-pregnant women [30,53-57].

Eicosapentanoic acid (EPA), one of n-3 PUFAs is significant for psychological health. It is mainly/mostly in fish and other sea foods [19]. EPA is a main constructive component of phospholipid membrane and is incorporated into cell membranes in whole body. It determines the biophysical characteristics of membrane of neurons[58]. Fatty acids affect receptors functions, neurotransmitters uptake, and signal transport. Association between EPA, inflammation and vascular disease may have a role in pathogenesis of depression because depression is associated with inflammation and atherosclerosis [59-61]. EPA is a precursor of some prostaglandins and Leukotrienes that are strong vasodilators and inhibitors of platelets aggregation and could decline the risk of vascular diseases [62]. Considering that docosehexaenoic acid (DHA) is essential for normal function of brain and EPA is a significant factor to inhibit inflammation process, a hypothesis poses that increase of fat acids N3 results in improvement of PPD. $1 \mathrm{~g} / \mathrm{d}$ of EPA has been shown to be an effective adjuvant to antidepressant therapy in resistant depressed patients [63-65]. Peet and Horrobin (2002) determined that addition of low-dose EPA to standard antidepressant therapy in depressed patients achieved a $50 \%$ improvement compared to a paraffin placebo with minimal side effects[63]. Therefore, addition of EPA supplementation may be beneficial for the $30-50 \%$ of patients resistant to standard depression treatment.

This study indicated that level of iron, iodine, folate, selenium, EPA was lower in hypochondriac participants. Unfortunately, there is no similar study to be compared with the findings of present study. Considering that depression and hypochondriac have significant association [7], deficiency of micronutrients related to hypochondriac or depression could aggravate each other. The present study has some strong points including population-based design, large sample size, and little drop-out rate (only 5 participants) that could prevent selection bias. Also, the study was conducted in whole rural areas of the Hormozgan province and was not limited to some special areas; since, depression and especially nutrition are associated with various factors such as socioeconomic status, education level, and culture that all of them could be influenced by the place of residence (rural or urban). Conducting the study in whole rural areas of the province prevented selection bias and yield a more precise assessment of association of nutrition, PPD and hypochondriasis. Moreover, in this study, potential confounding factors 
were controlled. For example, nutrition questionnaire was completed in each trimester (not after birth); so, the findings could not be influenced by appetite in various periods of pregnancy. Another strong point was using validated FFQ to assess nutritional status; since, it is a standard tool for assessing long-term nutritional status in cohort and cross-sectional studies. The limitation of present study was applying selfreported questionnaires for post-partum depression (Edinburg) and hypochondriasis. However, both questionnaires are used for screening as valid tools $[66,67]$.

\section{Conclusions}

The findings of present study showed that low intake of some micronutrients such as iron, selenium, iodine, folate, vitamin C, B12, EPA (in all trimesters) and vitamin c (in second trimester) are associated with PPD. Also, low intake of some micronutrients including iron, iodine, folate, selenium, EPA (in all trimesters) is associated with hypochondriasis. Nutrition is a controllable effective factor for hypochondriasis and PPD as high prevalence disorders with adverse outcomes. So, it is suggested that prenatal care providers put emphasize on consumption of micronutrients during pregnancy.

\section{Declarations}

\section{Data Availability Statement}

The primary data for this study is available from the authors (Fatemeh Bazarganipour) on direct request.

\section{Disclosure statement}

The authors report no conflicts of interest.

\section{Authors' contributions}

FB contributed in conception, design, statistical analysis and drafting of the manuscript. SA, ZA, MA and TD contributed in data collection and manuscript drafting. All authors approved the final version for submission. FB supervised the study.

\section{Funding}

Hormozgan University of Medical Science, Bandar-Abbas, Iran.

\section{Conflict of interest}

The authors declare that they have no conflict of interest. 


\section{Informed consent}

Informed consent was obtained from all individual participants included in the study.

\section{Ethical approval}

All procedures performed in studies involving human participants were in accordance with the ethical standards of the institutional and/or national research committee and with the 1964 Helsinki declaration and its later amendments or comparable ethical standards.

\section{References}

1. Eiris J, Novo-Rodriguez MI, Del Rio M, Meseguer P, Del Rio MC, Castro-Gago M. The effects on lipid and apolipoprotein serum levels of long-term carbamazepine, valproic acid and phenobarbital therapy in children with epilepsy. Epilepsy Res. 2000;41:1-7.

2. Yonkers KA, Vigod S, Ross LE. Diagnosis, pathophysiology, and management of mood disorders in pregnant and postpartum women. Obstet Gynecol. 2011;117:961-77. doi:10.1097/AOG.0b013e31821187a7.

3. Dennis CL, Chung-Lee L. Postpartum depression help-seeking barriers and maternal treatment preferences: a qualitative systematic review. Birth. 2006;33:323-31. doi:10.1111/j.523536X.2006.00130.x.

4. McGill HC Jr, McMahan CA, Herderick EE, Malcom GT, Tracy RE, Strong JP. Origin of atherosclerosis in childhood and adolescence. Am J Clin Nutr. 2000;72:1307S-15S.

5. de Chadarevian JP, Legido A, Miles DK, Katsetos CD. Epilepsy, atherosclerosis, myocardial infarction, and carbamazepine. J Child Neurol. 2003;18:150-1.

6. Schwind J, Neng JMB, Höfling V, Weck F. Health Behavior in Hypochondriasis. J Nerv Ment Dis. 2015;203:493-8.

7. H K, H G, H N, S T, A G. Depression and its relationship with hypochondriasis in nurses in Neyshabur, Iran. Military Caring Sciences. 2016;3:34-40.

8. Scarella TM, Laferton JAC, Ahern DK, Fallon BA, Barsky A. The Relationship of Hypochondriasis to Anxiety, Depressive, and Somatoform Disorders. Psychosomatics. 2016;57:200-7.

9. Lal J, Vasudev K, Kela AK, Jain SK. Effect of oral magnesium supplementation on the lipid profile and blood glucose of patients with type 2 diabetes mellitus. J Assoc Physicians India. 2003;51:3742.

10. Sockol LE, Epperson CN, Barber JP. Preventing postpartum depression: a meta-analytic review. Clin Psychol Rev. 2013;33:1205-17. doi:10.016/j.cpr.2013.10.004. Epub Oct 21.

11. Leung BM, Kaplan BJ. Perinatal depression: prevalence, risks, and the nutrition link-a review of the literature. J Am Diet Assoc. 2009;109:1566-75. doi:10.016/j.jada.2009.06.368. 
12. $10.3389 /$ fpubh. 2017.00093

Van Vo T, Hoa TKD, Hoang TD. Postpartum Depressive Symptoms and Associated Factors in Married Women: A Cross-sectional Study in Danang City, Vietnam. Front Public Health. 2017;5:93.:10.3389/fpubh.2017.00093. eCollection 2017.

13. Ding T, Wang DX, Qu Y, Chen Q, Zhu SN. Epidural labor analgesia is associated with a decreased risk of postpartum depression: a prospective cohort study. Anesth Analg. 2014;119:383-92. doi:10.1213/ANE.0000000000000107.

14. Aishwarya S, Rajendiren S, Kattimani S, Dhiman P, Haritha S, Ananthanarayanan PH. Homocysteine and serotonin: association with postpartum depression. Asian J Psychiatr. 2013;6:473-7. doi:10.1016/j.ajp.2013.05.007. Epub Jun 14.

15. Chi $X$, Zhang P, Wu H, Wang J. Screening for Postpartum Depression and Associated Factors Among Women in China: A Cross-Sectional Study. Front Psychol. 2016;7:1668. 10.3389/fpsyg.2016.01668. eCollection 2016.

16. Deng AW, Xiong RB, Jiang TT, Luo YP, Chen WZ. Prevalence and risk factors of postpartum depression in a population-based sample of women in Tangxia Community, Guangzhou. Asian Pac J Trop Med. 2014;7:244-9. doi:10.1016/S995-7645(14)60030-4.

17. Lewis SJ, Araya R, Leary S, Smith GD, Ness A. Folic acid supplementation during pregnancy may protect against depression 21 months after pregnancy, an effect modified by MTHFR C677T genotype. Eur J Clin Nutr. 2012;66:97-103. doi:10.1038/ejcn.2011.136. Epub Jul 20.

18. Rechenberg K, Humphries D. Nutritional interventions in depression and perinatal depression. Yale J Biol Med. 2013;86:127-37. Print 2013 Jun.

19. Bodnar LM, Wisner KL. Nutrition and depression: implications for improving mental health among childbearing-aged women. Biol Psychiatry. 2005;58:679-85.

20. Panwar B, Punia D. Food intake of rural pregnant women of Haryana State, northern India: relationship with education and income. Int J Food Sci Nutr. 1998;49:243-7.

21. Karimi Zarchi M, Behtash N, Chiti Z, Kargar S. Cervical cancer and HPV vaccines in developing countries. Asian Pac J Cancer Prev. 2009;10:969-74.

22. Ahmadi kani Golzar A, GoliZadeh Z. Validation of Edinburgh Postpartum Depression Scale (EPDS) for screening postpartum depression in Iran. J Nurs Educ. 2015;3:1-10.

23. Ren F, Feng W, Shi HR, Wu QH, Chen ZM. Value of frozen section examination in diagnosis and treatment of high-grade cervical intraepithelial neoplasia. Chin Med J (Engl). 2012;125:2462-5.

24. Fallucca F, Dalfra MG, Sciullo E, Masin M, Buongiorno AM, Napoli A, et al. Polymorphisms of insulin receptor substrate 1 and beta3-adrenergic receptor genes in gestational diabetes and normal pregnancy. Metabolism. 2006;55:1451-6.

25. Hosseini Esfahani F, Asghari G, Mirmiran P, Azizi F. Reproducibility and Relative Validity of Food Group Intake in a Food Frequency Questionnaire Developed for the Tehran Lipid and Glucose Study. Journal of Epidemiology. 2010;20:150-8. 
26. Beard J. Iron deficiency alters brain development and functioning. J Nutr. 2003;133:1468S. 72S. doi: 10.093/jn/133.5.S.

27. Iron deficiency-United States. 1999-2000. MMWR Morb Mortal Wkly Rep. 2002;51:897-9.

28. Onder G, Penninx BW, Cesari M, Bandinelli S, Lauretani F, Bartali B, et al. Anemia is associated with depression in older adults: results from the InCHIANTI study. J Gerontol A Biol Sci Med Sci. 2005;60:1168-72. doi:10.093/gerona/60.9.

29. Gosney MA, Hammond MF, Shenkin A, Allsup S. Effect of micronutrient supplementation on mood in nursing home residents. Gerontology. 2008;54:292-9. doi:10.1159/000131886. Epub 2008 May 8.

30. Mokhber N, Namjoo M, Tara F, Boskabadi H, Rayman MP, Ghayour-mobarhan M, et al. Effect of supplementation with selenium on postpartum depression: a randomized double-blind placebocontrolled trial. The Journal of Maternal-Fetal Neonatal Medicine. 2011;24:104-8.

31. Henley WN, Koehnle TJ. Thyroid hormones and the treatment of depression: an examination of basic hormonal actions in the mature mammalian brain. Synapse. 1997;27:36-44. doi: 10.1002/(SICI)982396(199709)27:1<36::AID-SYN4>3.0.CO;2-E.

32. Shor-Posner G, Lecusay R, Miguez MJ, Moreno-Black G, Zhang G, Rodriguez N, et al. Psychological burden in the era of HAART: impact of selenium therapy. Int J Psychiatry Med. 2003;33:55-69. doi:10.2190/PFFD-D920-V041-N5KD.

33. Moshrefi B, Delshad H, Karimzadeh L. Iodine Fortification Program Effectiveness: A Review on National Studies of lodine Fortification. Clinical Excellence. 2015;4:174-57.

34. Jin Y, Coad J, Weber JL, Thomson JS, Brough L. Selenium Intake in lodine-Deficient Pregnant and Breastfeeding Women in New Zealand. Nutrients. 2019;11:69.

35. Gulseren S, Gulseren L, Hekimsoy Z, Cetinay P, Ozen C, Tokatlioglu B. Depression, anxiety, healthrelated quality of life, and disability in patients with overt and subclinical thyroid dysfunction. Arch Med Res. 2006;37:133-9. doi:10.1016/j.arcmed.2005.05.008.

36. Guimaraes JM, de Souza Lopes C, Baima J, Sichieri R. Depression symptoms and hypothyroidism in a population-based study of middle-aged Brazilian women. J Affect Disord. 2009;117:120-3. doi:10.1016/j.jad.2008.12.012. Epub 9 Jan 25.

37. Bono G, Fancellu R, Blandini F, Santoro G, Mauri M. Cognitive and affective status in mild hypothyroidism and interactions with L-thyroxine treatment. Acta Neurol Scand. 2004;110:59-66. doi:10.1111/j.600-0404.2004.00262.x.

38. Baldini IM, Vita A, Mauri MC, Amodei V, Carrisi M, Bravin S, et al. Psychopathological and cognitive features in subclinical hypothyroidism. Prog Neuropsychopharmacol Biol Psychiatry. 1997;21:92535. doi:10.1016/s0278-5846(97)00089-4.

39. Ge JF, Peng YY, Qi CC, Chen FH, Zhou JN. Depression-like behavior in subclinical hypothyroidism rat induced by hemi-thyroid electrocauterization. Endocrine. 2014;45:430-8. doi:10.1007/s12020-0130001-4. Epub 2013 Jun 21.

40. Bodnar LM, Wisner KL. Nutrition and depression: implications for improving mental health among childbearing-aged women. Biol Psychiatry. 2005;58:679-85. doi:10.1016/j.biopsych.2005.05.009. 
Epub Jul 25.

41. Bottiglieri T, Folate. vitamin B12, and neuropsychiatric disorders. Nutr Rev. 1996;54:382-90. doi:10.1111/j.753-4887.1996.tb03851.x.

42. Chanarin I, Deacon R, Lumb M, Muir M, Perry J. Cobalamin-folate interrelations: a critical review. Blood. 1985;66:479-89.

43. Nafar M, Khatami F, Kardavani B, Farjad R, Pour-Reza-Gholi F, Firouzan A, et al. Role of folic acid in atherosclerosis after kidney transplant: a double-blind, randomized, placebo-controlled clinical trial. Exp Clin Transplant. 2009;7:33-9.

44. Marcucci R, Zanazzi M, Bertoni E, Rosati A, Fedi S, Lenti M, et al. Vitamin supplementation reduces the progression of atherosclerosis in hyperhomocysteinemic renal-transplant recipients. Transplantation. 2003;75:1551-5.

45. Landon MB, Gabbe SG. Gestational diabetes mellitus. Obstet Gynecol. 2011;118:1379-93.

46. Gilbody S, Lightfoot T, Sheldon T. Is low folate a risk factor for depression? A meta-analysis and exploration of heterogeneity. J Epidemiol Community Health. 2007;61:631-7. doi:10.1136/jech.2006.050385.

47. Papakostas GI, Petersen T, Mischoulon D, Ryan JL, Nierenberg AA, Bottiglieri T, et al. Serum folate, vitamin B12, and homocysteine in major depressive disorder, Part 1: predictors of clinical response in fluoxetine-resistant depression. J Clin Psychiatry. 2004;65:1090-5. doi:10.4088/jcp.v65n0810.

48. Alpert M, Silva RR, Pouget ER. Prediction of treatment response in geriatric depression from baseline folate level: interaction with an SSRI or a tricyclic antidepressant. J Clin Psychopharmacol. 2003;23:309-13. doi:10.1097/01.jcp.0000084024.22282.cd.

49. Coppen A, Bailey J. Enhancement of the antidepressant action of fluoxetine by folic acid: a randomised, placebo controlled trial. J Affect Disord. 2000;60:121-30. doi:10.1016/s0165327(00)00153-1.

50. Alpert JE, Mischoulon D, Rubenstein GE, Bottonari K, Nierenberg AA, Fava M. Folinic acid (Leucovorin) as an adjunctive treatment for SSRI-refractory depression. Ann Clin Psychiatry. 2002;14:33-8.

51. Resler G, Lavie R, Campos J, Mata S, Urbina M, Garcia A, et al. Effect of folic acid combined with fluoxetine in patients with major depression on plasma homocysteine and vitamin B12, and serotonin levels in lymphocytes. Neuroimmunomodulation. 2008;15:145-52. doi:10.1159/000151527. Epub 2008 Aug 21.

52. Coppen A, Bolander-Gouaille C. Treatment of depression: time to consider folic acid and vitamin B12. J Psychopharmacol. 2005;19:59-65. doi:10.1177/0269881105048899.

53. Jacka FN, Overland S, Stewart R, Tell GS, Bjelland I, Mykletun A. Association Between Magnesium Intake and Depression and Anxiety in Community-Dwelling Adults: The Hordaland Health Study. Australian \& New Zealand Journal of Psychiatry. 2009;43:45-52.

54. Pasco JA, Jacka FN, Williams LJ, Evans-Cleverdon M, Brennan SL, Kotowicz MA, et al. Dietary selenium and major depression: a nested case-control study. Complementary Therapies in Medicine. 
2012;20:119-23.

55. Sánchez-Villegas A, Doreste J, Schlatter J, Pla J, Bes-Rastrollo M, Martínez-González MA. Association between folate, vitamin B6 and vitamin B12 intake and depression in the SUN cohort study. Journal of Human Nutrition Dietetics. 2009;22:122-33.

56. Skarupski KA, Tangney C, Li H, Ouyang B, Evans DA, Morris MC. Longitudinal association of vitamin B-6, folate, and vitamin B-12 with depressive symptoms among older adults over time. The American Journal of Clinical Nutrition. 2010;92:330-5.

57. Smith KJ, Sanderson K, McNaughton SA, Gall SL, Dwyer T, Venn AJ. Longitudinal Associations Between Fish Consumption and Depression in Young Adults. Am J Epidemiol. 2014;179:1228-35.

58. Salem N Jr, Litman B, Kim HY, Gawrisch K. Mechanisms of action of docosahexaenoic acid in the nervous system. Lipids. 2001;36:945-59. doi:10.1007/s11745-001-0805-6.

59. Alexopoulos GS, Meyers BS, Young RC, Campbell S, Silbersweig D, Charlson M. 'Vascular depression' hypothesis. Arch Gen Psychiatry. 1997;54:915-22. doi:10.1001/archpsyc.997.01830220033006.

60. Sauter JL, Powlovich L, St John T, Leiman G. Have the 2006 American Society for Colposcopy and Cervical Pathology (ASCCP) Guidelines Enriched the Yield of High-Grade Squamous Intraepithelial lesions? Diagn Cytopathol. 2014.

61. Maes M, Smith R, Christophe A, Cosyns P, Desnyder R, Meltzer H. Fatty acid composition in major depression: decreased omega 3 fractions in cholesteryl esters and increased C20: 4 omega 6/C20:5 omega 3 ratio in cholesteryl esters and phospholipids. J Affect Disord. 1996;38:35-46. doi:10.1016/0165-327(95)00092-5.

62. Simon JA, Hodgkins ML, Browner WS, Neuhaus JM, Bernert JT Jr, Hulley SB. Serum fatty acids and the risk of coronary heart disease. Am J Epidemiol. 1995;142:469-76. doi:10.1093/oxfordjournals.aje.a117662.

63. Peet M, Horrobin DF. A dose-ranging study of the effects of ethyl-eicosapentaenoate in patients with ongoing depression despite apparently adequate treatment with standard drugs. Arch Gen Psychiatry. 2002;59:913-9. doi:10.1001/archpsyc.59.10.913.

64. Nemets B, Stahl Z, Belmaker RH. Addition of omega-3 fatty acid to maintenance medication treatment for recurrent unipolar depressive disorder. Am J Psychiatry. 2002;159:477-9. doi:10.1176/appi.ajp.159.3.477.

65. Frangou S, Lewis M, McCrone P. Efficacy of ethyl-eicosapentaenoic acid in bipolar depression: randomised double-blind placebo-controlled study. Br J Psychiatry. 2006;188:46-50. 10.1192/bjp.188.1.46.

66. Jardri R, Pelta J, Maron M, Thomas P, Delion P, Codaccioni X, et al. Predictive validation study of the Edinburgh Postnatal Depression Scale in the first week after delivery and risk analysis for postnatal depression. J Affect Disord. 2006;93:169-76. doi:10.1016/j.jad.2006.03.009. Epub Apr 27.

67. Bunevicius A, Kusminskas L, Pop VJ, Pedersen CA, Bunevicius R. Screening for antenatal depression with the Edinburgh Depression Scale. J Psychosom Obstet Gynaecol. 2009;30:238-43. doi:10.3109/01674820903230708. 